\title{
Cueing task goals and earning money: Relatively high monetary rewards reduce failures to act on goals in a Stroop task
}

\author{
Harm Veling • Henk Aarts
}

Published online: 30 March 2010

(C) The Author(s) 2010. This article is published with open access at Springerlink.com

\begin{abstract}
We examined the role of monetary rewards in failures to act on goals in a Stroop task. Based on recent developments in theorizing on the interplay between rewards and cognitive control, we hypothesized that relatively high monetary rewards enhance the focus and stability of a cued task goal compared to low monetary rewards, and hence cause a reduction in failures to act on current task goals under circumstances that warrant top-down goal implementation. To test this, participants received a modified version of the Stroop task, in which they were either briefly cued with the goal of naming the color or meaning of targets on a trial-bytrial basis. After goal cuing, but before presenting the target, either a low or high reward cue was presented. Results showed that higher rewards produced a general speed-up. More importantly, Stroop interference on error rates was lower in the high reward condition compared to the low reward condition, revealing that the rewards enhanced focus and stability of the cued goal. These results provide support for theorizing that reward processing modulates utility assessment of current goals by affecting attention to facilitate goal-directed behavior.
\end{abstract}

Keywords Reward · Goals · Cognitive control · Stroop interference

\section{Introduction}

In everyday life, people's goals can materialize quite briefly, rapidly and spontaneously. Keeping one's eye on

H. Veling $(\bowtie) \cdot H$. Aarts

Department of Psychology, Utrecht University, PO Box 80.140, 3508 TC Utrecht, The Netherlands e-mail: h.veling@uu.nl goals that are brought to mind quickly and suddenly is essential for effectively dealing with environmental demands, especially when multiple goals are potentially relevant, and current goals are not supported by bottom-up processes, but should be implemented by the cognitive system in a top-down fashion in the subsequent encounter of the environment. For example, perception of an empty glass elicits different goal-directed behaviors depending on whether ones goal is to serve a guest, or to do the dishes. Accordingly, in order to act effectively under such circumstances, it is essential to keep the currently relevant goal in mind (Miller and Cohen 2001).

In contemporary approaches to the cognitive control of goal-directed behavior a goal is conceived of as a mental representation of an intention to accomplish a task (e.g., Altmann and Trafton 2002; Hommel et al. 2001; Jeannerod 1997; Kornblum et al. 1990). An important question in this domain is how an individual's attention is focused on a relevant goal to stabilize its representation when there are other goals salient that should be ignored, and the situation provides no clues about what to do. In other words, the question is how the cognitive control of relevant goals is regulated. In the present research we examine the role of monetary rewards in this regulation. Specifically, we hypothesize that relatively high rewards cause people to more strongly focus on currently relevant goals. This focus should be especially helpful to facilitate goal-directed behavior in situations where one's goals cannot be derived from the environment, and hence, goals are more prone to forgetting. We report a study designed to test this intriguing possibility.

A paradigm that has been extensively used in research to examine the performance of cognitive control of goals is the Stroop (1935) task. In this task participants have the task goal to report the color in which words or word-like 
stimuli are presented as fast and accurately as possible. When the color and the meaning of the word are incongruent (the word blue presented in red), acting on the task goal of naming the color is slower, or more error prone, compared to when the color is congruent (the word blue presented in blue), or neutral to the word (e.g., XXXXX presented in blue; for a review see MacLeod 1991). Importantly, this Stroop interference effect is generally viewed as the result of two independent mechanisms (e.g., De Jong et al. 1999; Kane and Engle 2003; MacDonald et al. 2000).

Firstly, in order to react appropriately to an incongruent trial an individual has to overcome the pre-potent response bias to read the word instead of naming the color of the word (i.e., resolve a response conflict). More difficulty to overcome this response conflict is reflected in more Stroop interference (slower responses to incongruent trials than to congruent or neutral trials). More important for the present discussion, the second mechanism involves the extent to which the person focuses attention on the task and thus keeps the task goal in mind (i.e., naming the color of stimuli instead of reading them). Inadequately focusing attention on the goal and stabilizing its representation leads to forgetting and greater difficulty to act in line with the task goal, again reflected in more Stroop interference (particularly on error rates; Kane and Engle 2003; see also Jostmann and Koole 2007).

Here, we aim to show that relatively high monetary rewards pertaining to the implementation of two alternating cued task goals (i.e., of indicating the meaning or the color of a stimulus word) may partly overcome failures of acting on goals as a result of enhanced focused attention and stability of goals. In fact, the representation of cued goals typically shows a rapid decay of activation over very short periods of time, usually within a second, unless some intervention based on the utility of the goal focuses the attention to the goal (see also e.g., Altmann and Trafton 2002; Baddeley and Logie 1999). Research in neuroscience indicates that the connected pathways in subcortical areas (e.g., ventral striatum) and prefrontal cortex (PFC) may play a prominent role in the process of failures to act on currently relevant goals (cf. Miller and Cohen 2001; Cohen et al. 2002). Although the neurological basis is not yet fully delineated, it appears that the utility assessment of rewards attached to a goal releases norepinephrine in the locus coeruleus, thereby enhancing focused attention processes in the PFC and rendering the representation of the goal more stable.

Capitalizing on this notion, there is research to suggest that reward cues accompanying a cued goal to engage in specific behavior causes people to keep better focus on that goal (Aarts et al. 2008a, b; Bijleveld et al. 2009). For instance, short presentation of a goal in concert with positive reward renders people more successful in implementing the goal in response to subsequent goal-relevant opportunities, even after a delay longer than a few seconds. Goals that were presented without reward cues did not show this effect (Aarts et al. 2008a, b). Thus, although these findings are not conclusive, they suggest that rewards that are linked to a cued goal facilitate attention processes in the service of the goal, such that the goal can be more effectively implemented in a top-down way. Building on, and extending this previous work on the role of rewards in modulating the focus and stability of goals, we hypothesize that relatively high monetary rewards that accompany the cueing of a goal will decrease Stroop interference by decreasing failures to act on the goal, particularly in situations where the goal cannot be derived from the environment (i.e., on incongruent Stroop trials).

To examine our hypothesis, we presented participants with a modified version of the Stroop task (see Fig. 1; see e.g. MacDonald et al. 2000). In this task participants were cued alternately with the task goal to name the meaning of words, and the task goal to name the colors in which words are presented. Thus, which of the two goals is valid was unknown to participants until the goal was cued at the beginning of a trial. After cueing of one of the two task goals, but before the target word appeared, a coin of either 1 euro cent or 50 euro cent was briefly shown, and participants were told that they could win the money by acting on the cued goal quickly and accurately. The coin was

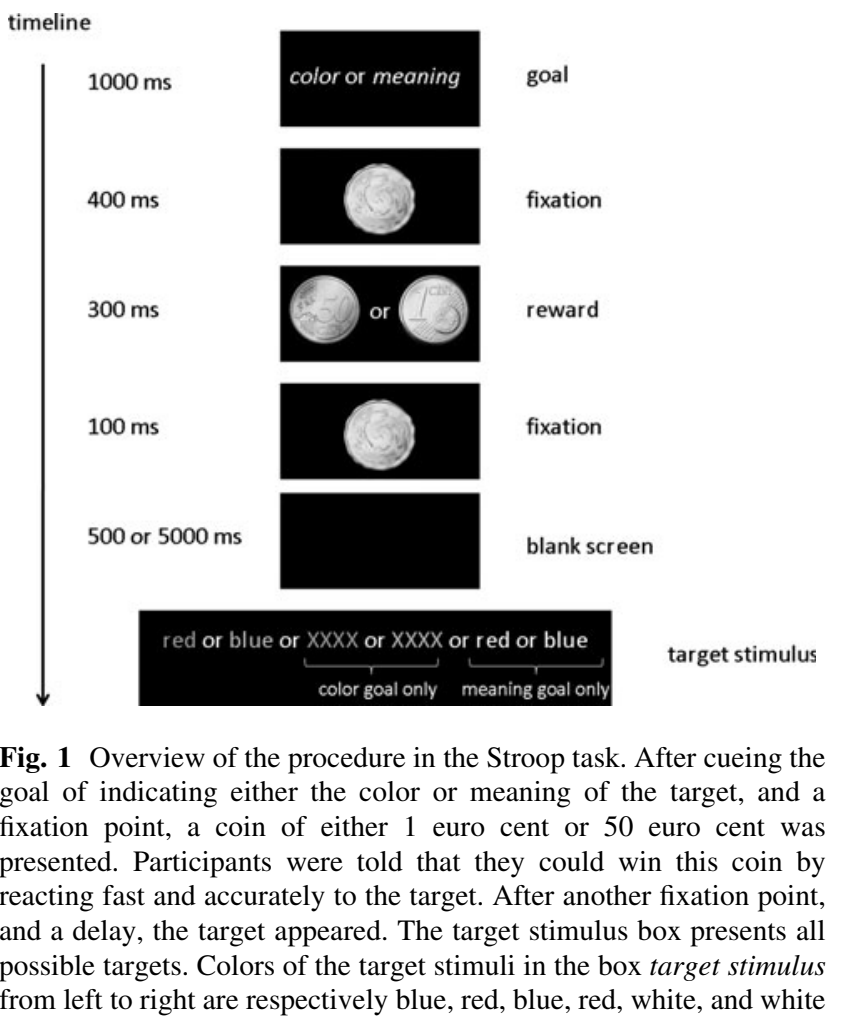


presented after cueing of the goal to avoid differences in encoding of the goal, and hence differences in baseline attention of the goals at the beginning of the trial. On half of the trials the Goal to Target Delay (GTD; i.e., the time between goal encoding and responding to the target) was set on 2,300 ms. This time interval was assumed to cause the activation of the goal representation to decay, and hence increased attention processes are required to keep focus on the goal over the critical period of time. This allowed us to examine the effects of monetary rewards of different value. For exploratory reasons, we also included trials of a GTD of $6,800 \mathrm{~ms}$ to examine whether cued goals that were treated with high (versus low) rewards would even survive a longer delay. Thus, the demands to put the cued goal into action were considerably high, as there were multiple potentially relevant goals (indicating the color or the meaning of the word) that were only cued just before one needed to implement them, and one needed to keep focus on the goal over time. Moreover, the different timings rendered the presentation of the target stimulus unpredictable.

Next, an incongruent or neutral target stimulus was presented (see Fig. 1). On incongruent trials the word meaning of the target stimulus was always inconsistent with its color (e.g., the word blue displayed in red). Note that this target stimulus contains conflicting stimulus dimensions for both the goal to indicate the meaning, and the goal to indicate the color. More importantly, also note that on these incongruent trials the previously cued goal cannot be derived from the target stimulus, so that accurate responding on such trials indicates that participants kept focus on the cued goal. Hence, any enhanced focus and stability of the cued goal representation by relatively high rewards should increase accuracy on incongruent trials.

On the other trials the target stimulus was neutral. In the case of the goal to indicate the meaning of the target these neutral trials consisted of the word blue or the word red always displayed in white color. In the case of the goal to indicate the color of the target these neutral trials always consisted of XXXXX displayed in either blue or red color. Note that on these neutral trials the goal-relevant stimulus dimension (color or meaning) was presented, but not the dimension of the other (conflicting) goal. Hence, on neutral trials the previously cued goal could be derived from the target stimulus (i.e., $\mathrm{XXXXX}$ in red or blue $=$ color goal; word red or blue presented in white $=$ meaning goal). Consequently, any enhanced focus and stability of the cued goal by relatively high rewards should not necessarily promote accuracy on neutral trials.

To test our hypothesis that relatively high rewards increase focus and stability of cued goal representations we examined both reaction times and error rates. On reaction times we expected two main effects. Firstly, we expected to find slower responses on incongruent trials than on neutral trials as a result of the (typical) occurrence of response conflicts on incongruent trials. Secondly, if relatively high rewards increase focus and stability of cued goals, participants should be more ready to implement goal congruent action (i.e., ready to respond to either the meaning or color of targets) once the target stimulus appears. Consequently, on relatively high reward trials we expected faster responses on both neutral and incongruent trials. Finally, note that even though we predict that increased focus on cued goals may be especially helpful when a target stimulus provides no information about what to do (i.e., on incongruent trials), increased performance on these trials compared to neutral trials may be difficult to observe on response latencies, as responses on neutral trials may also get faster by increased focus on cued goals.

Importantly, on error rates we expected an interaction between reward and congruency. Specifically, as the incongruent trials provide no information about which goal was cued (and neutral trials do), any increased focus and stability of cued goal representations should reduce error rates on incongruent trials, but not on neutral trials. Accordingly, compared to the 1 cent condition, we expected that the 50 cent condition would lead to lower Stroop interference, i.e., mean error rate on incongruent trials minus mean error rate on neutral trials, due to enhanced focus and stability of the cued goal. This predicted effect would suggest that relatively high compared to low rewards increase focus on cued task goals that require action soon.

\section{Method}

Participants and design

The experiment included 36 undergraduates ( 24 females). We employed a 2(reward: 1 cent vs. 50 cent) by 2(goal: indicate meaning vs. indicate color) by 2 (congruency: neutral vs. incongruent) by 2 (GTD: $2,300 \mathrm{~ms}$ vs. $6,800 \mathrm{~ms}$ ) within participants design.

\section{Stroop task}

The Stroop task consisted of 32 practice trials and 128 experimental trials. The procedure within each trial is depicted in Fig. 1. All stimuli within a trial were presented in the middle of a computer screen against a black background. On each trial, participants were first cued with a task goal. Specifically, when participants had the goal to indicate the meaning of a later presented target stimulus they were presented with the single word meaning for $1,000 \mathrm{~ms}$, and when they had the goal to indicate the color 
in which the target stimulus was displayed they were presented with the single word color for 1,000 ms (see e.g., MacDonald et al. 2000; for a similar goal cueing procedure). The goal cueing event was followed by a picture of a scrambled coin for $400 \mathrm{~ms}$. Next, a coin of either 1 euro cent or 50 euro cent was presented for $300 \mathrm{~ms}$, followed by another picture of a scrambled coin for $400 \mathrm{~ms}$. (see Bijleveld et al. 2009, 2010; for the effectiveness of this monetary reward presentation procedure). Then, the coin was followed by a blank screenshot, and finally the target stimulus. The duration of the blank screenshot varied, i.e. $500 \mathrm{~ms}$ on one half of the trials and 5,000 $\mathrm{ms}$ on the other half of the trials, such that the GTD was either 2,300 or $6,800 \mathrm{~ms}$. The target stimulus remained on the screen until participants pressed the A button to indicate blue or the $\mathrm{L}$ button to indicate red on a QWERTY keyboard.

On $50 \%$ of the trials the meaning of the target stimulus was inconsistent with its color, i.e. incongruent trials, and on the other $50 \%$ of the trials the target stimulus was neutral. As explained above, when the goal was to indicate the meaning of the stimulus these neutral trials consisted of the words blue or red displayed in white color. Furthermore, when the goal was to indicate the color of the stimulus the neutral trials consisted of $\mathrm{XXXXX}$ displayed in either blue or red color. We presented 8 trials in each cell of our 2(reward: 1 cent vs. 50 cent) by 2(goal: indicate meaning vs. indicate color) by 2(congruency: neutral vs. incongruent) by 2(GTD: relatively short vs. long) design, resulting in a total of 128 trials. In the practice phase participants received 2 trials of each trial type, resulting in 32 practice trials. Trials were presented in a random order.

\section{Procedure}

Participants were tested individually. They received instructions for the Stroop task, and read that they could win money by responding fast and accurately. They read that each trial would start with a $1,000 \mathrm{~ms}$ presentation of the task goal at issue: When the word color would be presented the task was to indicate the color of targets, and when the word meaning would be presented the task was to indicate the meaning of targets. Participants read that after this cueing of the task goal the amount to be won on the current trial would be presented, and that they would learn after the task how much extra money they had actually won. After performing the Stroop task they were debriefed about the nature of the study, and thanked and paid for their participation. Debriefing revealed that none of the participants indicated that their accuracy performance between the incongruent and neutral trials would differ as a function of the rewards. Thus, if different effects occur between incongruent and neutral trials as a function of rewards they seem to be rather implicit and subtle.

\section{Results}

Response latencies

To reduce the impact of incidental outliers on response latencies, and normalize the data, we omitted trials with responses faster than $300 \mathrm{~ms}$ and slower than $3,000 \mathrm{~ms}$ (.7\% of the data). Furthermore, we conducted analyses on log-transformed response latencies (Jostmann and Koole 2007), and report untransformed means for clarity. Nonetheless, analyses of the raw data lead to similar results.

Then, we performed the 2(reward: 1 cent vs. 50 cent) by 2(goal: indicate meaning vs. indicate color) by 2(congruency: neutral vs. incongruent) by 2(GTD: 2,300 vs. $6,800 \mathrm{~ms}$ ) within subjects analysis of variance (ANOVA) on response latencies. Because GTD did not interact with our reward manipulation $(F \mathrm{~s}<1.5$ for all interactions involving reward and GTD), we collapsed subsequent analyses over this factor. This analyses revealed a main effect of congruency, revealing the standard Stroop effect, $F(1, \quad 35)=118.80, \quad p<.01, \quad \eta_{\mathrm{p}}^{2}=.78, \quad$ (i.e., $\quad$ slower response latencies on incongruent trials compared with neutral trials; see Table 1). We also obtained the expected main effect of reward, $F(1,35)=5.04, p<.05, \eta_{\mathrm{p}}^{2}=.13$. As can be seen in Table 1, participants responded faster in the 50 cent condition than in the 1 cent condition. Although the raw means suggest that the Stroop effect might be weaker in the 50 cent condition compared to the 1 cent condition, reward and congruency did not interact, $F<1$. Finally, there was a main effect of goal, $F(1,35)=7.78$, $p<.01, \eta_{\mathrm{p}}^{2}=.18$, indicating that participants were faster to act on the goal to indicate the color $(M=776$; $\mathrm{SD}=233$ ) compared to acting on the goal to indicate the meaning $(M=811 ; \mathrm{SD}=237)$. No other effects on response latencies were found.

\section{Error rates}

Next, we performed the same 2(reward: 1 cent vs. 50 cent) by 2(goal: indicate meaning vs. indicate color) by 2(congruency: neutral vs. incongruent) by 2(GTD: 2,300 vs. $6,800 \mathrm{~ms}$ ) within subjects analysis of variance (ANOVA) on error rates. Because GTD did not interact with our reward manipulation ( $F_{\mathrm{S}}<1$ for all interactions involving reward and GTD), we again collapsed subsequent analyses over this factor. Firstly, this analysis revealed a main effect of congruency, $F(1,35)=64.13, p<.01, \eta_{\mathrm{p}}^{2}=.65$, which was qualified by an interaction with reward, $F(1$, $35)=7.47, p<.05, \eta_{\mathrm{p}}^{2}=.18$. As can be seen in Table 1 , and as predicted, the Stroop effect on error rates was reduced in the 50 cent condition compared to the 1 cent condition. Most importantly, participants made fewer errors on incongruent trials in the 50 cent condition than in 
Table 1 Mean response latencies and error rates as a function of reward and trial type

\begin{tabular}{|c|c|c|c|c|c|c|}
\hline \multirow[t]{3}{*}{ Reward } & \multicolumn{6}{|l|}{ Trial type } \\
\hline & \multicolumn{2}{|l|}{ Neutral } & \multicolumn{2}{|c|}{ Incongruent } & \multicolumn{2}{|l|}{ SI } \\
\hline & Latency & Error rate & Latency & Error rate & Latency & Error rate \\
\hline 1 cent & 720 (204) & $.02(.04)$ & 919 (330) & $.12(.09)$ & 199 (168) & $.10(.08)$ \\
\hline 50 cent & 705 (223) & $.04(.05)$ & 877 (292) & $.09(.07)$ & 171 (129) & $.05(.06)$ \\
\hline
\end{tabular}

Standard deviations are in parentheses. SI Stroop interference (incongruent - neutral)

the 1 cent condition, $F(1,35)=4.16, p<.05, \eta_{\mathrm{p}}^{2}=.11$. Unexpectedly, on neutral trials participants made more errors in the 50 cent condition than in the 1 cent condition, $F(1,35)=7.11, p<.05, \eta_{\mathrm{p}}^{2}=.17$. Possibly, in addition to enhanced focus on task goals, relatively high rewards increased eagerness to respond quickly (e.g., Bijleveld et al. 2010) which resulted in incidental errors. These incidental errors may also have occurred on incongruent trials, but as increased focus on the cued goal also reduced commission of errors on these trials the end result is less errors on incongruent trials in the relatively high reward condition compared to the low reward condition. Therefore, the reduction in Stroop interference in the relatively high reward condition may be the best estimate of the degree to which relatively high rewards increased focus on cued task goals. Finally, there was a main effect of goal, $F(1,35)=8.39, p<.01, \eta_{\mathrm{p}}^{2}=.19$, indicating that error rates were larger in the color goal condition $(M=.08$; $\mathrm{SD}=.07)$ compared to the meaning goal condition $(M=.05 ; \mathrm{SD}=.05)$. No other effects on error rates were found.

\section{Discussion}

In the present research we examined whether monetary rewards can reduce failures to act on a cued task goal that is not further supported by bottom-up processes. Firstly, and as predicted, relatively high rewards led to overall faster responses. This overall speed-up is consistent with the hypothesis that high compared to low rewards increase focus on current goals, as increased focus should facilitate goal implementation when the opportunity arises. More importantly, results further showed that high rewards led to less commission of errors when targets were uninformative about which goal should be applied (i.e., on incongruent trials) compared to when the goal could be derived from the targets (i.e., on neutral trials). Because we cued the goals before presenting the reward information, this reduction in Stroop interference on error rates cannot be attributed to increased attention to the goals when they were cued. Instead, this finding suggests that relatively high rewards increased focus on the cued goal that was already active in memory, thus preparing the cognitive system to implement the goal in a top-down fashion. Once the opportunity arose to act on the goal, this increased focus reduced the error rate on incongruent trials compared to neutral trials. This result converges well with current models of cognitive control that assume that the utility assessment of rewards attached to a goal enhances focused attention processes rendering the representation of the goal more stable (Cohen et al. 2002). What we add here is the important insight that such enhanced attention to specific task goals as a function of rewards has beneficial effects especially when the goal is not supported by bottom-up processes via the environment (e.g., incongruent vs. neutral targets in a Stroop task).

Although the faster responses and reduced Stroop interference on error rates in the relatively high reward condition are consistent with an increased focus interpretation, one could argue that these findings may also be explained in more general terms. That is, one could argue that relatively high rewards may function as a kind of call for increased effort to perform well (Bijleveld et al. 2009; Locke and Braver 2008; Pochon et al. 2002; Waugh and Gotlib 2008). In case of the faster response latencies such a general effort explanation may indeed be applicable. Specifically, relatively high rewards may also increase eagerness to respond quickly to the target stimuli, resulting in overall faster responses. This general explanation is not appropriate to explain reduced Stroop interference on error rates, however, as fewer errors on incongruent trials compared to neutral trials specifically indicates that relatively high rewards increased the likelihood that the cued goal was kept in mind. Thus, although relatively high rewards may increase motivation and effort to perform well, possibly reflected by a general effect on task performance (an overall speed-up of responses), they also increased focus on recently cued goals as revealed by a relative reduction in erroneous goal implementation when the environment did not provide any clues about what to do. This suggests that rewards in the present research promote goal-directed behavior through two routes: (1) a more general one that increases the overall motivation and effort, and (2) a more 
strategic one that increases attention and stability of the specific task goal at hand.

The present results suggest that relatively high rewards did not facilitate response conflict resolution (i.e., as in that case relatively high rewards should have especially led to faster responding to incongruent trials compared to neutral trials; Kane and Engle 2003; Mcfall et al. 2009). Interestingly, though, other work has shown that increased effort, induced by social evaluation, can reduce Stroop interference on response latencies (Mcfall et al.; Experiment 2). It is important to note, however, that in this previous research a regular Stroop task was used in which there was only one goal (i.e., respond to the color of target stimuli). McFall et al., have argued that in this case more effort on incongruent trials can facilitate responding by stronger corrective efforts to override response conflicts. In the present research both color and meaning where randomly alternated and potentially relevant on a trial-by-trial basis, so that the task did not only involve resolving response conflicts on incongruent trials, but first and foremost required focusing on the cued goals. This rather salient task demand may have attenuated any effects of increased effort on the more subtle task demand to resolve response conflicts.

Notably, previous work on rewards and Stroop interference that examined the effects of monetary rewards at the general task level (i.e., perform well on the Stroop task) between participants, did not find reliable effects of this reward manipulation on Stroop interference (Huguet et al. 2004). A key difference between the current experiment and this previous research is that we used a modified version of the Stroop task that alternated between two goals on a trial-by-trial basis, which increased the need to have the relevant goal in mind for each trial separately, whereas a standard Stroop task (including one goal that of indicating the color) was used in earlier work. Furthermore, in the present experiment, we examined the contribution of rewards at the concrete goal level within participants, thus testing the on-line nature of rewards in focusing attention as to the specificity of the goal at hand. Rewards are likely most effective in improving focus and stability when they are presented in a context that involves multiple goals that are potentially relevant (cf. Cohen et al. 2002).

In the present research we compared the high reward condition with the low reward condition instead of a no reward condition. This procedure has been applied in a number of studies on the effects of monetary rewards on performance on different tasks (e.g., Bijleveld et al. 2009, 2010; Pessiglione et al. 2007). One advantage of this experimental set-up is that the control condition (i.e., low reward condition) remains very similar to the high reward condition, but differs only on one aspect (i.e., value). This was especially important in the present experiment where we presented the reward information after goal cueing, and it is likely that presentation of any information between goal cueing and target presentation will affect performance. Nonetheless it is an interesting question of whether the effects of high rewards depend on a relative comparison with the low rewards (e.g., 50 cents is relatively more than 1 cent), or whether it is the intrinsic value of the high reward (e.g., 50 cent) that gives rise to the current effects. This question provides a promising avenue for future research.

Recently, there have been a number of studies published that examined the interplay between goal activation and rewards (e.g., Aarts et al. 2008a, b; Custers and Aarts 2005; see also Bijleveld et al. 2010). This research has primarily focused on effects of rewards in affecting the incentive value of the cued goal, or persistence (effort or resource recruitment) to reach the end state of a goal. The present research extends previous work on the relation between goals and rewards by showing the facilitative effect of monetary rewards in focusing attention on cued task goal representations, resulting in improved goal-implementation when this implementation is not supported by bottom-up processes via the environment, but should be implemented by the cognitive system in a top-down fashion. A reward can thus function as a signal that promotes the significance of a specific goal in a specific context. By elucidating the role of rewards in increasing the focus and stability of goals that are briefly cued in people's mind, we believe that the present research shows one important function of rewards, and hope that more attention will be devoted to the role of rewards in goal-directed behavior and cognitive control in future research.

Acknowledgments We thank the Goallab Research Group for their helpful comments and feedback regarding the work reported in this manuscript. The work in this paper was financially supported by the Netherlands Organization for Scientific Research (NWO VICI-grant 453-06-002).

Open Access This article is distributed under the terms of the Creative Commons Attribution Noncommercial License which permits any noncommercial use, distribution, and reproduction in any medium, provided the original author(s) and source are credited.

\section{References}

Aarts, H., Custers, R., \& Marien, H. (2008a). Preparing and motivating behaviour outside of awareness. Science, 319, 1639.

Aarts, H., Custers, R., \& Veltkamp, M. (2008b). Goal priming and the affective-motivational route to nonconscious goal pursuit. Social Cognition, 26, 555-577.

Altmann, E. M., \& Trafton, J. G. (2002). Memory for goals: An activation-based model. Cognitive Science, 26, 39-83.

Baddeley, A. D., \& Logie, R. H. (1999). Working memory: The multiple-component model. In A. Miyake \& P. Shah (Eds.), Models of working memory: Mechanisms of active maintenance 
and executive control (pp. 28-61). New York, NY: Cambridge University Press.

Bijleveld, E., Custers, R., \& Aarts, H. (2009). The unconscious eyeopener: Pupil size reveals strategic recruitment of resources upon subliminal reward cues. Psychological Science, 20, 1313-1315.

Bijleveld, E., Custers, R., \& Aarts, H. (2010). Unconscious reward cues increase invested effort, but do not change speed-accuracy tradeoffs. Cognition, 115, 330-335.

Cohen, J. D., Braver, T. S., \& Brown, J. W. (2002). Computational perspectives on dopamine function in prefrontal cortex. Current Opinion in Neurobiology, 12, 223-229.

Custers, R., \& Aarts, H. (2005). Positive affect as implicit motivator: On the nonconscious operation of behavioral goals. Journal of Personality and Social Psychology, 89, 129-142.

De Jong, R., Berendsen, E., \& Cools, R. (1999). Goal neglect and inhibitory limitations: Dissociable causes of interference effects in conflict situations. Acta Psychologica, 101, 379-394.

Hommel, B., Müsseler, J., Aschersleben, G., \& Prinz, W. (2001). The theory of event coding (TEC): A framework for perception and action planning. Behavioral and Brain Sciences, 24, 849-878.

Huguet, P., Dumas, F., \& Monteil, J.-M. (2004). Competing for a desired reward in the Stroop task: When attentional control is unconscious but effective versus conscious but ineffective. Canadian Journal of Experimental Psychology, 58, 153-167.

Jeannerod, M. (1997). The cognitive neuroscience of action. Blackwell: Oxford.

Jostmann, N. B., \& Koole, S. L. (2007). On the regulation of cognitive control: Action orientation moderates the impact of high demands in Stroop interference tasks. Journal of Experimental Psychology: General, 136, 593-609.

Kane, M. J., \& Engle, R. W. (2003). Working-memory capacity and the control of attention: The contributions of goal neglect, response competition, and task set to Stroop interference. Journal of Experimental Psychology: General, 132, 47-70.
Kornblum, S., Hasbroucq, T., \& Osman, A. (1990). Dimensional overlap: Cognitive basis of stimulus-response compatibility-A model and taxonomy. Psychological Review, 97, 253-270.

Locke, H. S., \& Braver, T. S. (2008). Motivational influences on cognitive control: Behavior, brain activation, and individual differences. Cognitive, Affective, and Behavioral Neuroscience, $8,99-112$.

MacDonald, A. W., Cohen, J. D., Stenger, V. A., \& Carter, C. S. (2000). Dissociating the role of the dorsolateral prefrontal and anterior cingulate cortex in cognitive control. Science, 288, $1835-1838$.

MacLeod, C. M. (1991). Half a century of research on the Stroop effect: An integrative review. Psychological Bulletin, 109, 163203.

McFall, S., Jamieson, J., \& Harkins, S. (2009). Testing the mere effort account of the evaluation-performance relationship. Journal of Personality and Social Psychology, 96, 135-154.

Miller, E. K., \& Cohen, J. D. (2001). An integrative theory of prefrontal cortex function. Annual Review of Neuroscience, 24, 167-202.

Pessiglione, M., Schmidt, L., Draganski, B., Kalisch, R., Lau, H., Dolan, R. J., et al. (2007). How the brain translates money into force: A neuroimaging study of subliminal motivation. Science, 316, 904-906.

Pochon, J. B., Levy, R., Fossati, P., Lehericy, S., Poline, J. B., Pillon, B., et al. (2002). The neural system that bridges reward and cognition in humans: An fmri study. Proceedings in the National Academy of Sciences of the United States of America, 99, 5669-5674.

Stroop, J. R. (1935). Studies of interference in serial verbal reactions. Journal of Experimental Psychology, 18, 643-662.

Waugh, C. E., \& Gotlib, I. H. (2008). Motivation for reward as a function of required effort: Dissociating the 'liking' from the 'wanting' system in humans. Motivation and Emotion, 32, 323330. 